\title{
Rotating Electric Machine Thermal Study
}

\author{
A.-I. Chirilă ${ }^{1}$, C. Ghiţă ${ }^{1}$, A. Crăciunescu ${ }^{1}$, I.-D. Deaconu ${ }^{1}$, V. Năvrăpescu ${ }^{1}$ and M. Catrinoiu ${ }^{1}$ \\ ${ }^{1}$ Department of Electrical Engineering \\ University Politehnica of Bucharest \\ Splaiul Independenţei, nr. 313, Sector 6, Bucharest, (Romania) \\ Phone/Fax number: +40 402 9564, e-mail: aurel.chirila@upb.ro, ghita.constantin@gmail.com
}

\begin{abstract}
This paper presents a mathematical model and numerical simulation results for heat transfer analysis that takes place at the frame level of a rotating electric machines provided with longitudinal cooling fins. For this purpose a relatively complex three-dimensional model is used, numerical treatable with affordable hardware and software resources. The frameenvironment convective heat transfer process is considered. The frame is cooled by forced convection in two cases: the thermal radiation heat transfer is taken into account and without considering it. Numerical simulation results reveal the heat transfer pathways from the frame to the environment, and allow assessment of important parameters in designing the machine's cooling.
\end{abstract}

\section{Key words}

Heat transfer coefficient, electric machine, computation, finite element.

\section{Introduction}

Rotating electrical machines used in electric drive systems can operate in different duty cycles under normal or overload conditions. The main malfunctions that arise during operation of these machines are of electromagnetic, thermal or mechanical nature. From mechanical and thermal point of views, the electromagnetic stresses inside the machine's iron core and windings are chosen such that the machine to withstand. The degree of loading is utmost related to the thermal state of the machine, i.e. the heat. However, in accordance with the considered duty cycle and environment conditions the machine must operate under load for a long period of time, such that the temperature of any part is not greater than the insulation class threshold. For this reason the thermal regimes during machine's operation are studies of great importance.

Inside a machine the heat transfer occurs due to the thermal imbalance between two media. This transfer is by conduction, convection or radiation. Heat transfer always occurs in one direction, from warmer medium towards the coldest one, and it will end when the two temperatures become equal. The study is based on heat transfer principles of thermodynamics, conduction physics, heat conversion and thermal radiation. Analysis of thermal phenomena involves description by mathematical expressions between the phenomena specific quantities and auxiliary quantities directly related to the specific quantities.

The electric machines specific thermal issues are macroscopic. Their thermal regimes are characterized by heat sources together with other specific quantities such as thermal time constants, thermal coefficients of materials and of the environment geometry in which heat transfer takes place.

Thermal stresses of an electrical machine conclude the effective output that normally can be provided. Determination of heat distribution inside the machine is a difficult task that requires a large amount of calculation. Addressing this problem can be done by finite difference numerical methods [1], [2], finite element numerical methods [3], [4], [5] or by using equivalent thermal schemes (global analysis of machine) [6], [7]. All previously mentioned approaches require knowledge of thermal parameters of the machine components.

The thermal study of the machine is performed here by using the finite element method because it has the advantage of better modelling the geometries of various parts of the machine. Similar studies based on finite element method can be found in [8-11].

In the field of electrical drives, knowing the loading limits of a machine for various operating modes is an important issue because the output power must be such as to lead to its normal operation, knowing that both under-sizing and over-sizing of the machine are not acceptable solutions from economical point of view.

In most variable speed electric drive systems the electric motor is induction type. Usually, these machines have an IP44 ingress protection at least and are totally enclosed fan cooled type. The induction machines have a small airgap, such that the internal cooling is insufficient. For this reason, the machine is cooled using an external fan mounted on the machine's shaft. In order to increase the cooling efficiency the machine housing has longitudinal fins that increase the heat transfer rate towards the environment by increasing the housing's outer surface. 
Determining the heat transfer coefficients with an increased accuracy allows for an adequate estimation of the machine's heating for given environment's temperature, duty cycle and losses. In the technical literature are presented many experimental studies regarding the heat transfer coefficients identification [12][14].

This paper presents a mathematical model and numerical simulation results for heat transfer analysis that takes place between the finned housing of an electrical machine and the environment. It is used a fairly complex threedimensional model, numerical treatable on accessible hardware/software resources. It is considered the convective heat transfer mode, housing - environment. The considered case is forced convection with/without radiation heat transfer.

\section{Machine description and computation hypotheses}

To study the housing-environment heat transfer a totally enclosed fan cooled three-phase squirrel cage induction machine is considered. Its main parameters are [15]: rated power $3 \mathrm{~kW}$, synchronous speed $1500 \mathrm{rpm}$ and size 112 . The study is performed for a fixed amount of losses that are known from the no-load and locked-rotor tests. The hypotheses made for this study are: the machine is loaded such that the total losses (windings losses, iron cores losses) give a heat flux of about $3000 \mathrm{~W} / \mathrm{m} 2$ uniformly distributed on the inner surface of the frame; the real detailed geometry of the cooling fins on the frame is accounted for; the heat is evacuated towards the environment only through the outer surface of the frame.

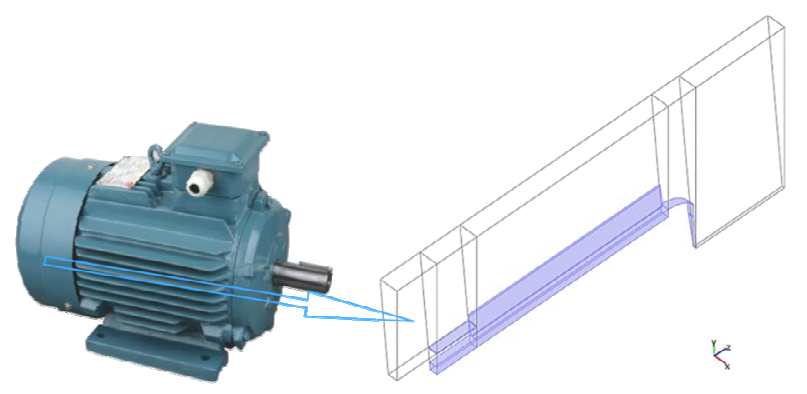

Fig. 1. Real and modelled geometry.

The assumed heat transfer modes are the thermal conduction between the inner surface and the outer surface of the housing, and the forced convection between the housing outer surface and environment. The external fan mounted on the shaft guides the cooling air stream between the cooling channels by means of the fan cowling (Fig. 1, left side). Because the airflow has a relatively high speed between $2 \mathrm{~m} / \mathrm{s}$ and $10 \mathrm{~m} / \mathrm{s}$, the achieved convection mode is forced convection. This means that natural convection can be neglected, and from this point of view is no longer important horizontal or vertical position of the machine on the test bench. For this reason the machine will be considered placed horizontally, as in Fig. 1. If the external fan of the machine was missing, heat transfer would be via natural convection and, in this case, the machine should be vertical so that the axes of the cooling channels defined by the housing fins are parallel to the gravity acceleration vector.

If forced convection, the temperature of the housing is relatively small, so in this analysis thermal radiation heat transfer is neglected. In forced convection, the cooling air is newtonian with constant thermal properties. The mediums have constant thermal properties also. The values are set based on the average temperature of the system.

Actual physical problem is three dimensional and its rigorous modelling should take into account the entire volume of the machine, so that the boundary conditions (flow, heat transfer) can be formulated as precisely. This approach is not practical because it leads to intensive hardware and software requirements. In consequence, the three dimensional model is cut down. Based on the symmetry of the cooling channels only one may be considered, defined by two consecutive fins. In order to still reduce the problem just half of the cooling channel is modelled. This has the advantage to be able to increase the mesh refinement in areas where quantities have important gradients and so a satisfying solving precision. Under these conditions, numerical solutions were obtained up to air inlet speed about $8 \mathrm{~m} / \mathrm{s}$. At very low air speeds (below $2 \mathrm{~m} / \mathrm{s}$ ) natural convection can not be neglected anymore.

\section{The mathematical model for thermal analysis}

The mathematical model developed for the steady flow with heat transfer problem is defined by the momentum balance (Navier-Stokes), the mass balance (continuity) and the energy balance. Specific constitutive relations are added to the model completeness [10]. Heat transfer processes are described by the following system of coupled nonlinear partial differential equations:

Momentum balance (Navier-Stokes)

$$
\rho(\boldsymbol{u} \cdot \nabla) \boldsymbol{u}=-\nabla p+\mu \nabla^{2} \boldsymbol{u}
$$

Mass balance (incompressible flow)

$$
\nabla \cdot \boldsymbol{u}=0
$$

Energy balance

$$
\rho c_{p}(\boldsymbol{u} \cdot \nabla) T=k \nabla^{2} T
$$

In eqs. (1)-(3) the following quantities are used: $u-$ velocity; $p$ - pressure; $T$ - temperature; $\mu$ - dynamic viscosity; $\rho$ - mass density; $k$ - thermal conductivity; $c_{p}-$ specific heat (at constant pressure). The heat generation within the fluid (by viscosity) is negligible - the main source of heat is considered on the boundary, at the boundary that represents the inner face of the housing. In Fig. 2 is presented the three dimensional computational model of a cooling channel defined by two successive fins. The cooling fins are assumed to have the same height equal to the majority of the real housing fins. The height 
differences of the fins around the housing are insignificant. The modelled cooling channel is only one half, i.e. the median vertical symmetry plane is used. This plane is perpendicular on the flowing direction.

The mathematical model was numerically implemented and solved in the COMSOL finite element (FEM) Multiphysics environment, based on Galerkin formulation [16]. The mathematical model can be implemented and solved using various dedicated software such as ANSYS CFX [17]. In [18] it is presented a benchmark showing that COMSOL is a bit faster than ANSYS in solving fluid dynamics problems but it requires more hardware (memory). In consequence the three dimensional model was solved using intensive resources, 32GB RAM memory. The central processing unit was made of 4 Dual Core processors.

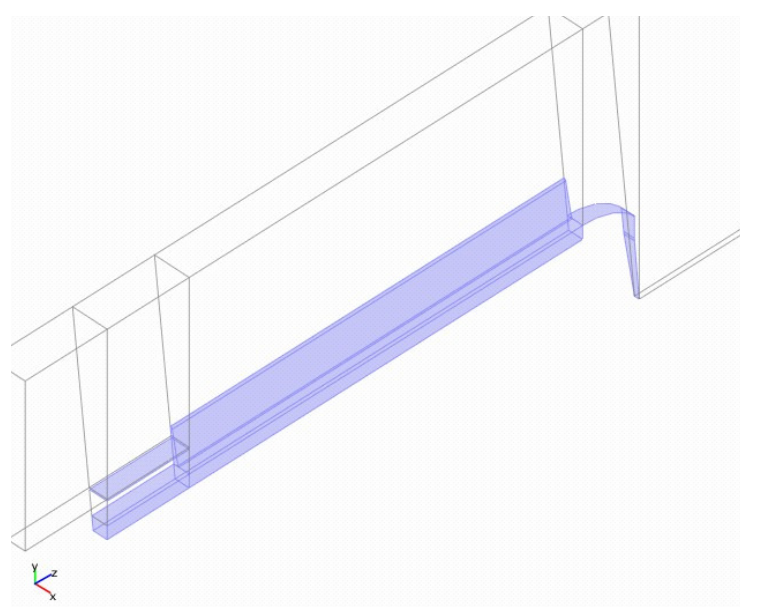

Fig. 2. The cooling channel.

The used unstructured mesh is presented in Fig. 3 and it contains about 400000 tetrahedral quadratic Lagrange elements. In order to obtained it, a double adaptation refinement algorithm has been applied, one for the heat problem and the second for the flow problem. To solve the algebraic system, a parallel direct solver was used.

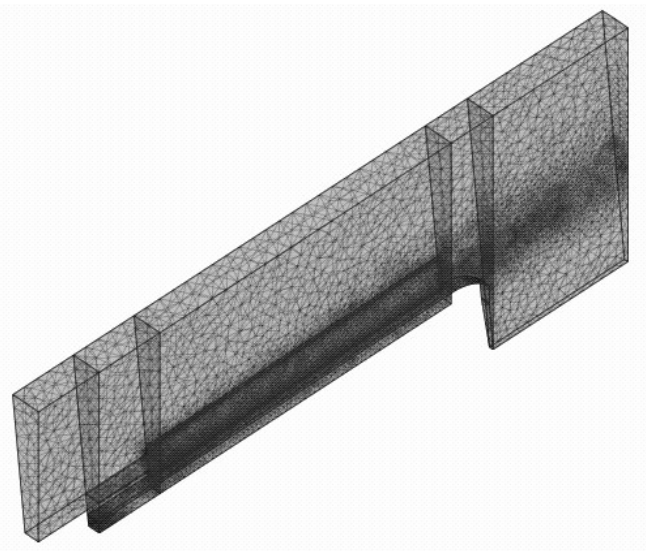

Fig. 3. Model mesh grid.

\section{Results without radiation}

Initially are presented the obtained results by taking into account only heat conduction and forced convection. The numerical solutions obtained by integrating the mathematical model shows both the paths of heat transfer and the cooling fluid flow around the housing outer surface. For example, in Fig. 4 is presented the result for an inlet speed of the cooling air, i.e. the air flow within the domain and the temperature field. During the flow, the fluid accumulates heat from the warm surface of the housing. At the outlet some air has a temperature close to the inlet air temperature, especially at the middle. This phenomenon can be explained by the fact that the air in the middle of the channel has a higher speed than the air near the surface. In other words, the air flowing through the cooling channel is similar to concentrated air jet form. This form appears due to the similarity with the plane Poiseuille flow [19], where the planes are defined by the channel walls, i.e. fins lateral sides. As the air progresses, it loses its effectiveness and so the housing becomes increasingly warmer.

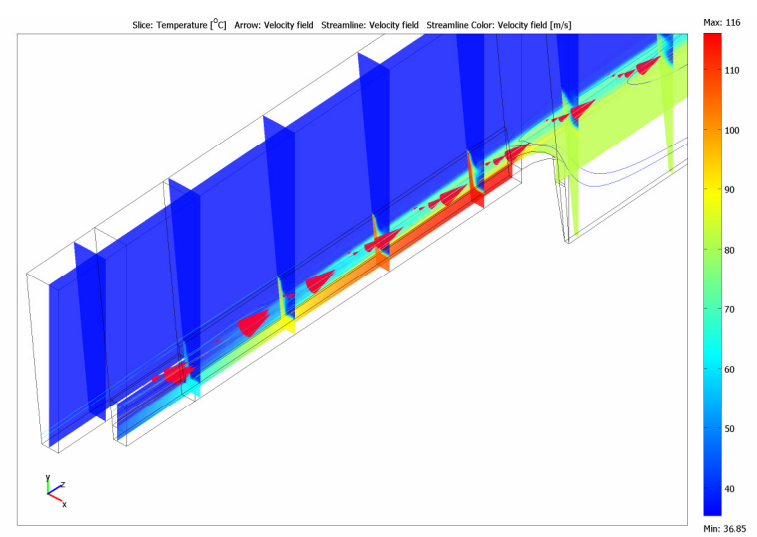

Fig. 4. Fluid flow and temperature map for air inlet velocity $\mathrm{u}=4 \mathrm{~m} / \mathrm{s}$.

For the thermal study an important quantity is the housing-environment heat transfer coefficient $h$. It indicates how much of the generated active power $P$ is transferred per unit area of the housing when there is a difference of one degree between surface temperature and ambient temperature. The mathematical expression of the coefficient is:

$$
h=\frac{P}{S \cdot \Delta T}=\frac{P}{S \cdot \theta_{S}}
$$

where $S$ is the surface, $\Delta T$ the temperature difference between the housing and the ambient, and $\theta_{S}$ is the surface over-temperature referred to the ambient temperature. 


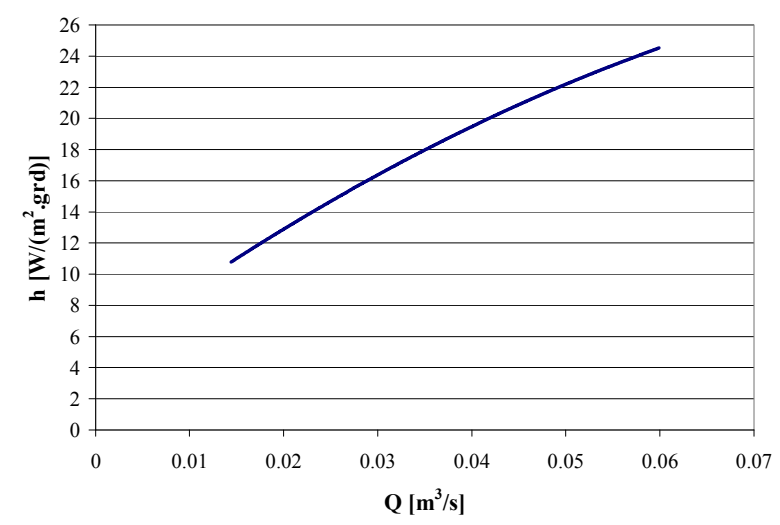

Fig. 5. Heat transfer coefficient vs. cooling airflow rate.

In Fig. 5 and Fig. 6 are shown the variations of the heat transfer coefficient with air flow and the average temperature variation with the air inlet speed respectively, both for no radiation forced convection case. In Fig. 6 it can be observed the saturation trend of the cooling efficiency with the increase of inlet air speed. This is explainable by the fact that the increase of air speed diminishes the air capability to accumulate heat.

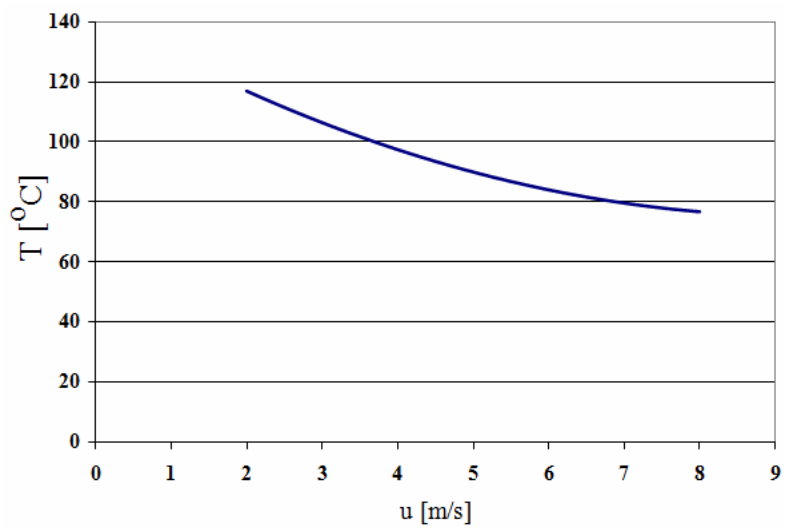

Fig. 6. Housing average temperature vs. inflow air speed.

\section{Results with radiation}

In order to account for radiation heat transfer the mathematical model is similar but supplementary a boundary condition was added. In this way the radiation heat flux is considered. To compare the results obtained in cases with radiation and no radiation the same inlet air velocity at the entrance to the channel, equal to $4 \mathrm{~m} / \mathrm{s}$ was chosen.

Regarding the airflow through the channel no significant difference is found. However, the average temperature of the housing is lower when the radiation is considered. The maximum difference obtained is about $9^{\circ} \mathrm{C}$. Thus, it can be concluded that the radiation is important for cooling the housing when the air velocity is low.

In Fig. 7 is presented a comparison of the heat transfer coefficient variation with the airflow in the cases of radiation and no radiation. When the radiation is not considered this coefficient is the forced convection heat transfer coefficient. In the other case this coefficient is a global heat transfer coefficient, i.e. radiation and convection, and in consequence it increases.

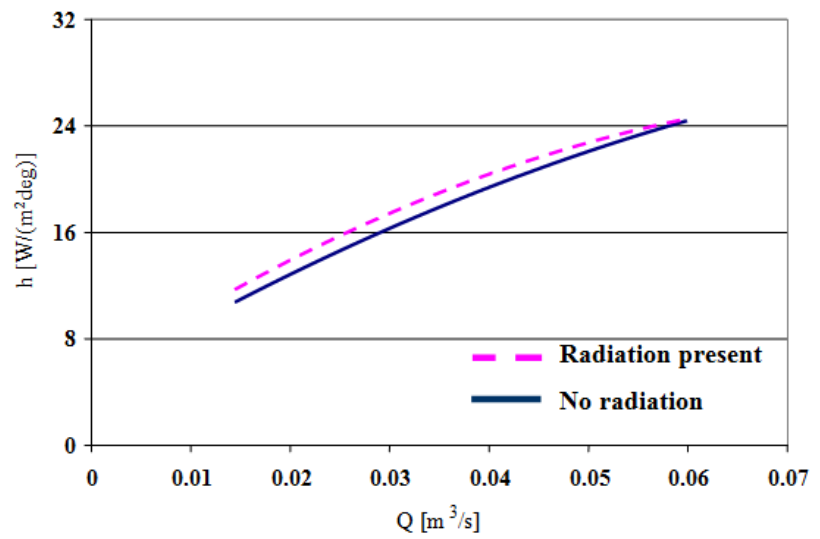

Fig. 7. Heat transfer coefficient vs. cooling airflow rate.

Similarly, in Fig. 8 it is shown the variation of housing average temperature versus inlet air velocity, with and without consideration of radiation. Hence it is concluded that with the increase of the inlet speed (air flow) the radiation influence over the housing temperature is diminishing.

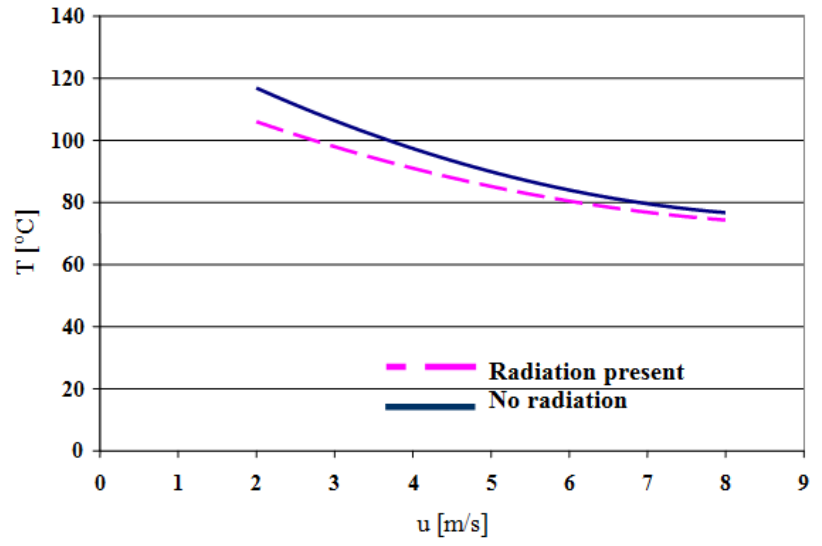

Fig. 8. Housing average temperature versus inlet air velocity.

\section{Conclusions}

The mathematical model for studying the thermal power machine is a complex one, because it includes the fluid flow and heat transfer. Its integration it is performed using finite element analysis that requires powerful software and hardware equipment. The three dimensional implemented model allows to finely reproduce all the details of the real geometry, i.e. the form of the housing fins, side shields, fan cowl.

The results are reflected in determination of the housingambient convection heat transfer coefficient when the radiation heat transfer is considered and not. Heat transfer coefficient is an essential parameter in the design of electric machines in terms of heat, especially to determine its power for different operating regimes. This coefficient can be used both for numerical methods (FEM) and the models with equivalent lumped thermal circuits. Knowing the heat transfer coefficient with a good precision helps to choose a fan that is optimal in terms of energy. It appears 
that the coefficient of convection does not change significantly, if one takes into account the phenomenon of thermal radiation.

The presented study heat can be applied to all types of electrical machines. What distinguishes these machines is the housing type (round or finned) and geometric structure of the channels or cooling fins.

\section{Acknowledgement}

The work has been co-funded by the Sectoral Operational Programme Human Resources Development 2007-2013 of the Romanian Ministry of Labour, Family and Social Protection through the Financial Agreement POSDRU/89/1.5/S/62557 and EDL - UPB.

\section{References}

[1] Hoffmann K.A., Chiang S.T., Computational Fluid Dynamics, Publishing house - Engineering Education System, 2000.

[2] Versteeg H.K., Malalasekera W., An introduction to Computational Fluid Dynamics - The Finite Volume Method, Editura Longman Scientific \& Technical, 1995.

[3] Lewis R.W., Nithiarasu P., Seetharamu K. N., Fundamentals of the Finite Element Method for Heat and Fluid Flow, Publishing house - John Wiley \& Sons, 2004.

[4] Shaw C.T., Using Computational Fluid Dynamics, Publishing house - Prentice Hall, 1992.

[5] Chung T. J., Computational Fluid Dynamics, 4th Edition, Vol. 1\&2, Publishing house - Cambridge University Press, 2002.

[6] Deaconu I.D., Ghiță C., Chirilă A.I., Năvrăpescu V., Popescu M., Thermal Study of Induction Machine using Motor-CAD, The $3^{\text {rd }}$ International Symposium on Electrical and Electronics Engineering (ISEEE'10), Galaţi, Romania, pg. 23-27, September $16-18,2010$.

[7] D.G. Dorrell, D.A. Staton, M.I. McGilp, "A Combined Electromagnetic and Thermal Approach to the Design of Electrical Machines", IEEE Industrial Electronics - IECON 2006, Paris, Nov 2006

[8] Lefik M., Komeza K., Computer modelling of $3 D$ transient thermal field coupled with electromagnetic field in one-phase induction motor with locked rotor, International Journal for Computation and Mathematics in Electrical and Electronic Engineering, 27, 4, pag. 861-868, 2008.

[9] Paladino E., Abdu E.A., Cezário C.A., Verardi M., Borges S.S., Improving Electric Motor Cooling System Efficiency, WEG Electric Motors, ANSYS Solutions, Fall, 2005.

[10] Masson P.J., Tixador P., Ordonez J.C., Morega A.M., Luongo C.A., Electro-Thermal Model for HTS Motor Design, IEEE Transactions on Applied Superconductivity, 17, 2, pag. 1529-1532, 2007.

[11] Chirilă A.I., Morega A.M, Electric motor frame cooling - A finite element analysis, The XXXIInd „Caius Iacob” National Conference on Fluid Mechanics and its Technical Applications, Oct. 16 - 17, Bucharest Romania, 2009.

[12] Alexeev, A.E., Construcţia maşinilor electrice, Editura Energetică de Stat, Bucureşti, 1954.

[13] W. Schuisky, Berechnung elektrischer Maschinen, Springer-Verlag, Wien, 1960

[14] Richter, R., Maşini Electrice, Editura Tehnică, Bucureşti, 1958

[15] Chirilă A.I., Theoretical and experimental approaches regarding the operation and optimization of induction machines within electrical drive systems, $\mathrm{PhD}$ Thesis, Politehnica University of Bucharest, 2010, Romania.
[16] COMSOL Multiphysics v.3.5a, Comsol A.B., Sweden, 2009.

[17] ANSYS Corporation - Fluid Dynamics Solutions. Available: http://www.ansys.com/products/fluid-dynamics/cfx/

[18] Wang X., Pepper D.W., Benchmarking COMSOL Multiphysics 3.4, Nevada Center for Advanced Computational Methods, Department of Mechanical Engineering, University of Nevada Las Vegas, 2007.

[19] Papanastasiou T.C., Georgiou G.C., Alexandrou A.N., Viscous Fluid Flow, Publishing house CRC Press, 2000. 